\title{
Microstructure and Tensile Properties of a Nb-Mo Microalloyed 6.5Mn Alloy Processed by Intercritical Annealing and Quenching and Partitioning
}

\author{
Ming-Hui Cai ${ }^{1} \cdot$ Hong-Shou Huang ${ }^{1} \cdot$ Hai-Jun Pan $^{1} \cdot$ Sheng-Hui Sun $^{1} \cdot$ Hua Ding ${ }^{1} \cdot$ Peter Hodgson $^{2}$
}

Received: 28 February 2017/Revised: 11 April 2017/Published online: 19 May 2017

(C) The Chinese Society for Metals and Springer-Verlag Berlin Heidelberg 2017

\begin{abstract}
The transformation behavior, microstructural evolution and mechanical properties were compared in a coldrolled $\mathrm{Nb}-\mathrm{Mo}$ microalloyed $6.5 \mathrm{Mn}$ alloy after intercritical annealing (IA) and quenching and partitioning (Q \& P), respectively. The thermodynamic calculation and theoretical analysis were used to determine the optimal heat treatment parameters. The Q \& P samples exhibited relatively higher strength with relatively low ductility, mainly due to the hard martensite matrix, which resulted in continuous yielding behavior upon loading, whereas the IA samples showed the significantly improved ductility, which benefited from the more sufficient transformation-induced plasticity (TRIP) effects and the softer ultrafine ferrite matrix. The dependence of yield point elongation (YPE) of IA samples on grain size demonstrated that the YPE value was in the reverse proportional relationship to the average grain size, which agreed well with theoretical analysis.
\end{abstract}

KEY WORDS: Medium Mn steel; Intercritical annealing; Quenching and partitioning; Mechanical properties; TRIP effect; Yield point elongation

\section{Introduction}

It is an inevitable trend to reduce the vehicle body structure weight in the modern automotive industry in order to improve fuel efficiency [1], for which one effective method is to use advanced high strength steel (AHSS). The current research on a new generation of automotive lightweight AHSS mainly involves: (1) the strength and toughness which are much higher than the first-generation AHSS without too many alloying elements; (2) the addition of

Available online at http://link.springer.com/journal/40195.

Ming-Hui Cai

cmhing@126.com

1 School of Materials Science and Engineering, Northeastern University, Shenyang 110819, China

2 Institute for Frontier Materials, Deakin University, Geelong, VIC 3216, Australia alloying elements which is much lower than the secondgeneration AHSS [2,3]. Medium manganese steel as a candidate of the third-generation AHSS family has received more attention due to its excellent comprehensive mechanical properties, and relatively low material and production costs [4-6]. Until now, the research on intercritical annealing (IA) process of medium Mn steels has been extensively carried out; however, the reports about the application of quenching and partitioning $(\mathrm{Q} \& \mathrm{P})$ process in medium Mn steels were limited.

In recent years, domestic and foreign scholars in materials and metallurgy have investigated the influences of alloying elements (e.g., $\mathrm{C}, \mathrm{Si}, \mathrm{Mn}, \mathrm{Al}$ ) and IA parameters on the microstructural evolution and mechanical properties of medium Mn steels [7, 8]. It has been demonstrated that the medium Mn steels treated by IA could typically possess such a microstructure, consisting of a large fraction of retained austenite $\left(\gamma_{R}\right)$, ultrafine ferrite and a small amount of martensite, which could dramatically improve the tensile ductility of materials without loss of tensile strength, when 
compared to the conventional transformation-induced plasticity (TRIP) steels. Studies on medium Mn steels have been well reviewed by Suh et al. [9], showing that medium Mn steels exhibited excellent products of ultimate tensile strength and total elongation, ranging from 21.0 to 65.6 $\mathrm{GPa} \%$. The good trade-off between strength and ductility is attributed in part to the extra work hardening produced by the TRIP effect which can occur in the volume fraction of $\gamma_{\mathrm{R}}$ as high as $30 \%[10,11]$.

The Q \& P process was firstly proposed by Speer et al. [12] as a new approach to produce the third-generation AHSS consisting of the carbon-rich $\gamma_{\mathrm{R}}$ and the carbondepleted martensite. The $\mathrm{Q} \& \mathrm{P}$ steels exhibited high strength and moderate ductility, as compared to the IA steels. The carbon constraint paraequilibrium (CCE) model was also proposed by Wang et al. and Speer et al. to predict the optimum quenching temperature (QT), where the maximum volume fraction of retained austenite $\left(V_{\mathrm{R}}\right)$ $[13,14]$ can be obtained. The "CCE" model performed well in predicting the $V_{\mathrm{R}}$ of $\mathrm{Q} \& \mathrm{P}$ steels with low alloying content.

Although the combined effects of $\mathrm{Nb}$ and Mo in the low carbon microalloyed steels (HSLAs) have been well documented $[15,16]$, there is little research on the role of $\mathrm{Nb}$ and Mo in medium Mn steels. In the present work therefore, the IA and $\mathrm{Q} \& \mathrm{P}$ approaches were designed and compared for the cold-rolled $\mathrm{Nb}-\mathrm{Mo}$ microalloyed medium Mn steels, based on our preliminary work [17]. The optimum annealing temperature (AT) during IA process and the QT during $\mathrm{Q} \& \mathrm{P}$ process were determined by means of thermodynamic calculation and theoretical analysis. Moreover, the influences of holding time and quenching temperature on the microstructural evolution and mechanical properties of IA and Q \& P steels were investigated, together with further analysis of transformation mechanism and yielding behavior during IA and Q \& $\mathrm{P}$ processes.

\section{Experimental Procedure}

\subsection{Materials and Its Preparation}

The $\mathrm{Nb}-\mathrm{Mo}$ microalloyed medium $\mathrm{Mn}$ steel with composition of $\mathrm{Fe}-0.16 \mathrm{C}-6.5 \mathrm{Mn}-1.1 \mathrm{Al}-0.05 \mathrm{~N}-0.22 \mathrm{Mo}-0.03 \mathrm{~N}$ (wt $\%$ ) was prepared in a laboratory vacuum induction furnace and cast into a $50 \mathrm{~kg}$ round ingot, followed by forging into a square billet. Subsequently, the billet was then homogenized at $1200{ }^{\circ} \mathrm{C}$ for $2 \mathrm{~h}$ with protection of $\mathrm{Ar}$ atmosphere, followed by oil quenching to room temperature after hot rolling to about $4.5 \mathrm{~mm}$ in thickness. The hotrolled plate was finally subjected to the multi-pass cold rolling by approximately $70 \%$ reduction.

\subsection{Design of Heat Treatment Processes}

The Q \& P process can be divided into three stages: the initial austenitizing stage, the quenching stage, and the final partitioning stage. They were performed in three different salt bath furnaces. The optimum QT and AT parameters were determined using the thermodynamic software JMatPro combined with theoretical calculation. For the $Q$ \& $\mathrm{P}$ process, the as-received cold-rolled samples were reheated to 800 and $850{ }^{\circ} \mathrm{C}$ for $15 \mathrm{~min}$ in a high-temperature salt bath furnace, quickly quenched in a low-temperature salt bath furnace of $170{ }^{\circ} \mathrm{C}$ for $20 \mathrm{~s}$, and then put in a middle-temperature salt bath furnace of $450{ }^{\circ} \mathrm{C}$ for $5 \mathrm{~min}$. The IA samples were first subjected to isothermal holding at an intercritical temperature $\left(650^{\circ} \mathrm{C}\right)$ for various holding times (10-360 $\mathrm{min}$ ) in an Ar protective atmosphere, followed by air cooling to room temperature. The schematic illustrations of two heat treatments are shown in Fig. 1a, b, respectively.

\subsection{Tensile Testing}

The samples for tensile tests were prepared with a cross section of $1 \mathrm{~mm} \times 10 \mathrm{~mm}$ and a gage length of $25 \mathrm{~mm}$, according to the ASTM E-8M sub-size standard [18]. The room temperature tensile tests were performed on a SANSCMT-5000 machine at a constant rate of $1.25 \mathrm{~mm} /$ $\min$.

\subsection{Microstructural Characterization}

The microstructural characterization of experimental steels was performed in a field emission scanning electron microscope (FE-SEM, ZEISS, $20 \mathrm{kV}$ ). Thin foils for transmission electron microscopy (TEM) observation were mechanically thinned to $50-60 \mu \mathrm{m}$, followed by electropolishing with a twin-jet electro-polisher in a solution of 95\% acetic acid and 5\% perchloric acid at a voltage of $45 \mathrm{~V}$ and about $16^{\circ} \mathrm{C}$. TEM observation was carried out on TECNAI-G ${ }^{2}-20$ operated at $120 \mathrm{kV}$. The X-ray diffraction (XRD) was used to determine the volume fraction of retained austenite $\left(V_{\mathrm{R}}\right)$. After proper electropolishing, the samples were scanned in the angle range of $40^{\circ}$ to $120^{\circ}$ with a speed of $2 \%$ min in a D/MAX-550 diffractometer with $\mathrm{Cu} K \alpha$ radiation. In the present work, the integrated intensities of (200) $\alpha$, (211) $\alpha$, (200) $\gamma$ and (220) $\gamma$ diffraction peaks were used to calculate the $V_{R}$ using the following equation [19].

$$
V_{\mathrm{R}}=\left(\frac{1}{q} \sum_{j=1}^{q} \frac{I_{\gamma j}}{R_{\gamma j}}\right) /\left[\left(\frac{1}{q} \sum_{j=1}^{q} \frac{I_{\gamma j}}{R_{\gamma j}}\right)+\left(\frac{1}{p} \sum_{i=1}^{p} \frac{I_{\alpha i}}{R_{\alpha i}}\right)\right],
$$


(a)

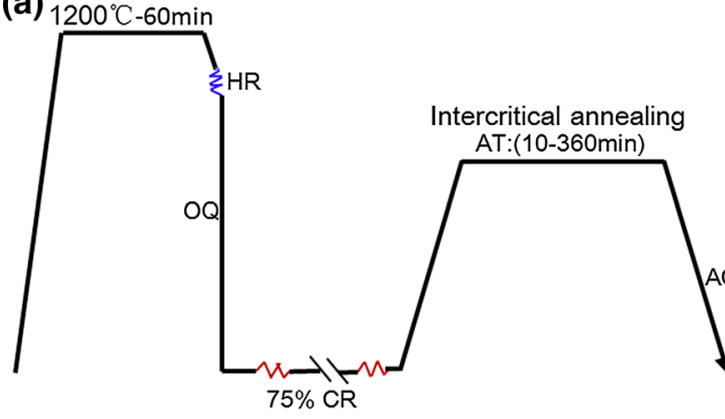

(b)

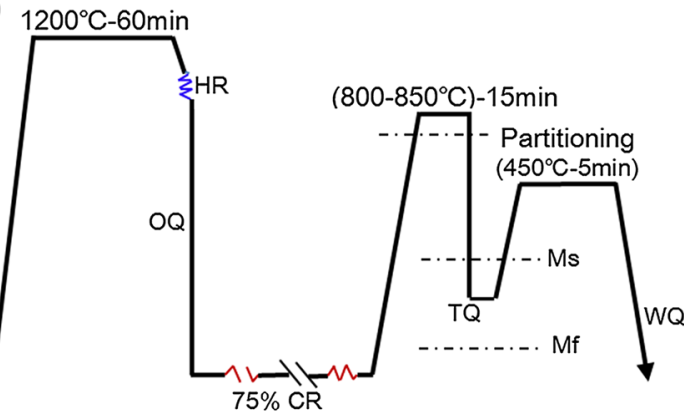

Fig. 1 Schematic illustration of heat treatment procedures of the cold-rolled Nb-Mo microalloyed 6.5Mn steel: a IA process; b Q \& P process

where $I_{\gamma i}$ and $R_{\gamma j}$ are the measured integrated intensities and the theoretical integrated intensities of $\gamma_{R}$, respectively; $I_{\alpha i}$ and $R_{\alpha j}$ are the measured integrated intensities and the theoretical integrated intensities of ferrite or martensite, respectively.

\section{Experimental Results}

\subsection{Optimization of Process Parameters}

It is known that the excellent mechanical properties of medium Mn steels are mainly attributed to the TRIP effect of retained austenite upon deformation [20, 21], so it is necessary to predict the maximum $V_{\mathrm{R}}$ through empirical formulas to obtain the optimal process parameters.

\subsubsection{Determination of Optimal AT for IA Process}

According to the authors' previous work [17], the maximum $V_{\mathrm{R}}(\sim 35 \%)$ of the experimental steel was obtained at approximately $650{ }^{\circ} \mathrm{C}$, which was selected in the present work as the optimal annealing temperature during IA process.

\subsubsection{Determination of Optimum $Q T$ for $Q \& P$ Process}

Seo et al. [22] have verified the diffusion of Mn element from martensite to austenite during $\mathrm{Q} \& \mathrm{P}$ process of the $0.2 \mathrm{C}-4.0 \mathrm{Mn}$ steel using $3 \mathrm{D}$ atom probe tomography (APT); however, the distance of Mn diffusion was quite short (only $\sim 2 \mathrm{~nm}$ ). In this case, it can be assumed that the effect of the diffusion of Mn atoms could be neglected for the calculation of optimal QT during $\mathrm{Q} \& \mathrm{P}$ process. Therefore, it is reasonable to determine the maximum $V_{\mathrm{R}}$ of medium Mn Q \& P steels using "CCE" model. Considering the sensitivity of $V_{\mathrm{R}}$ to the QT [23], so the optimal QT during Q \& $\mathrm{P}$ process was determined as follows.

The volume fraction of austenite to martensite can be predicted using the following equation [24].
$V_{\mathrm{M}}=1-\exp \left[-K_{\mathrm{LV}} \times\left(M_{\mathrm{s}}-T\right)^{n_{\mathrm{LV}}}\right]$,

where $V_{\mathrm{M}}$ is the volume fraction of martensite, $T$ is the quenching temperature, $K_{\mathrm{LV}}$ and $n_{\mathrm{LV}}$ are coefficients, which mainly depend on the chemical compositions.

The coefficient $K_{\mathrm{LV}}$ and $n_{\mathrm{LV}}$ in Eq. (2) can be calculated as follows.

$$
\left\{\begin{aligned}
K_{\mathrm{LV}}= & 0.0231-0.0105 \mathrm{C}-0.0017 \mathrm{Ni} \\
& +0.0074 \mathrm{Cr}-0.0193 \mathrm{Mo} \\
n_{\mathrm{LV}}= & 1.4304-1.1836 \mathrm{C}+0.7527 \mathrm{C}^{2} \\
& -0.0258 \mathrm{Ni}-0.0739 \mathrm{Cr}+0.3108 \mathrm{Mo}
\end{aligned}\right.
$$

where the amount of each element is percent in weight.

The $M_{\mathrm{s}}$ equation that was proposed by Capdevila et al. [25] was widely used to predict the martensite transformation start temperature for low alloy steels. However, the $M_{\mathrm{s}}$ of the present steel was predicted to be about $242{ }^{\circ} \mathrm{C}$, which was relatively higher than that $\left(\sim 200{ }^{\circ} \mathrm{C}\right)$ obtained by the JMatPro software. Thus, the modified $M_{\mathrm{s}}$ equation was expressed as:

$$
\begin{aligned}
M_{\mathrm{S}}\left({ }^{\circ} \mathrm{C}\right)= & 491.2-302.6 \mathrm{C}-30.6 \mathrm{Mn}-14.5 \mathrm{Si}-16.6 \mathrm{Ni} \\
& -8.9 \mathrm{Cr}+2.4 \mathrm{Mo}-11.3 \mathrm{Cu}+7.4 \mathrm{~W}-42 .
\end{aligned}
$$

According to the "CCE" model, the change in the predicted phase fractions with QT is shown in Fig. 2. The solid bold curve represents the variation of $V_{\mathrm{R}}$ as a function of QT. It can be seen that the final $V_{\mathrm{R}}$ first increased and then decreased with increasing the QT. Thus, the optimal QT was determined to be about $170{ }^{\circ} \mathrm{C}$, at which the corresponding maximum $V_{\mathrm{R}}$ was about $20 \%$.

\subsection{Evolution of Microstructures}

\subsubsection{Evolution of Microstructures for $Q \& P$ Steels}

The as-cold-rolled microstructure by $75 \%$ reduction showed a typical broken lath martensite structure with dozens of nanometers in width, as illustrated in Fig. 3a. 
Figure $3 b$, c shows the SEM micrographs of the $\mathrm{Q} \& \mathrm{P}$ samples austenitized for $15 \mathrm{~min}$ at 800 and $850{ }^{\circ} \mathrm{C}$, respectively. It can be seen that under both austenitizing conditions, the microstructures exhibited the tempered lath martensite, which was characterized by indistinctive grain boundaries. In contrast, the lath of martensite is relatively longer and wider when the samples were austenitized at $850{ }^{\circ} \mathrm{C}$ rather than $800{ }^{\circ} \mathrm{C}$, due to the coarse primary austenite grains at $850{ }^{\circ} \mathrm{C}$.

TEM was used to identify the fine microstructures of the $\mathrm{Q} \& \mathrm{P}$ processed $\mathrm{Nb}-\mathrm{Mo}$ microalloyed $6.5 \mathrm{Mn}$ steel, as displayed in Fig. 4. It can be found that the film-like $\gamma_{R}$ (40-50 nm in width) can be observed in both samples as seen in Fig. 4a, e. For the sample austenitized at $800{ }^{\circ} \mathrm{C}$, both the lath-like martensite $(\sim 200 \mathrm{~nm}$ in width) and twinning martensite (Fig. 4c) were observed, whereas for the sample austenitized at $850{ }^{\circ} \mathrm{C}$ no twinning martensite

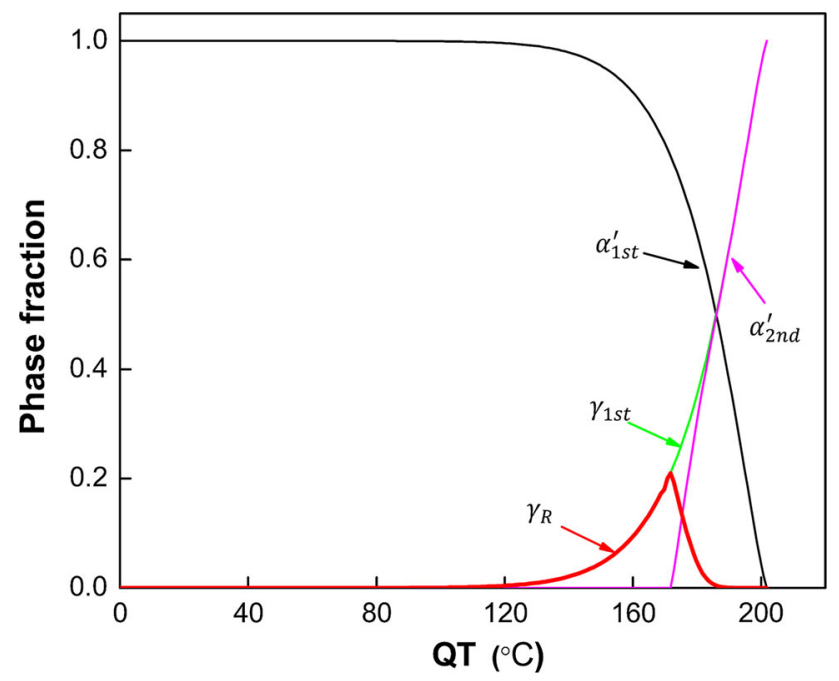

Fig. 2 Predicted variation in different phase fractions as a function of QT for the cold-rolled Nb-Mo microalloyed 6.5Mn steel treated by Q $\&$ P: $\gamma_{\mathrm{R}}, \gamma_{1 s t}, \alpha_{1 s t}^{\prime}$ and $\alpha_{2 \text { nd }}^{\prime}$, which represent the final retained austenite, austenite after initial quenching, primary martensite and secondary martensite, respectively appeared and the width of lath martensite was slightly larger, approximately 200-300 nm. It should be also noted that some amount of carbide-free bainite with a very fine film shape ( $\sim 10 \mathrm{~nm}$ in width) was observed in the sample austenitized at $850{ }^{\circ} \mathrm{C}$, as arrowed in Fig. 4d.

The volume fraction of $V_{\mathrm{R}}$ of the $\mathrm{Q} \& \mathrm{P} \mathrm{Nb}-\mathrm{Mo}$ microalloyed $6.5 \mathrm{Mn}$ steel was evaluated using $\mathrm{XRD}$, as shown in Fig. 5. It can be seen that both bcc and fcc diffraction peaks can be detected, and the $V_{\mathrm{R}}$ of samples austenitized at $800{ }^{\circ} \mathrm{C}(\sim 12.1 \%)$ is slightly higher than that of samples austenitized at $850{ }^{\circ} \mathrm{C}(\sim 11.1 \%)$.

\subsubsection{Evolution of Microstructures for IA Steels}

Figure 6 shows the SEM micrographs of the cold-rolled $\mathrm{Nb}-\mathrm{Mo}$ microalloyed $6.5 \mathrm{Mn}$ steel after IA at $650{ }^{\circ} \mathrm{C}$ for different holding time, ranging from 10 to $360 \mathrm{~min}$. Regardless of holding time, the microstructures mainly consisted of equiaxial ferrite $(\alpha)$ and $\gamma_{\mathrm{R}}$ : (1) as $t<30 \mathrm{~min}$, the grain size of both phases reached ultrafine and nanoscale level; (2) as $t<180 \mathrm{~min}$, the grain size reached the sub-micron level; (3) as $t<360 \mathrm{~min}$, the grain size reached the fine-grained level. Moreover, the stacking fault (SF) was also observed to some extent when the samples were annealed for more than $270 \mathrm{~min}$ (Fig. 6e, f), as demonstrated by TEM, which indicates that the austenite in some local areas had low stacking fault energy because of variation of the $\mathrm{Mn}$ and/or carbon concentration as a function of annealing time.

Figure $7 \mathrm{a}$ is the XRD patterns before and after IA processes at $650{ }^{\circ} \mathrm{C}$ for different holding time, showing that only bcc patterns were found in the as-cold-rolled samples, while both fcc and bcc diffraction peaks existed in the IA samples. Figure $7 \mathrm{~b}$ shows the variation of $V_{\mathrm{R}}$ as a function of annealing time. It can be found that the $V_{\mathrm{R}}$ first increased and then decreased with increasing the annealing time. The maximum value was obtained at $30 \mathrm{~min}$, approximately $23 \%$. It is also worth noting that the decreasing trend of $V_{\mathrm{R}}$ was slight as the annealing time beyond $60 \mathrm{~min}$. The
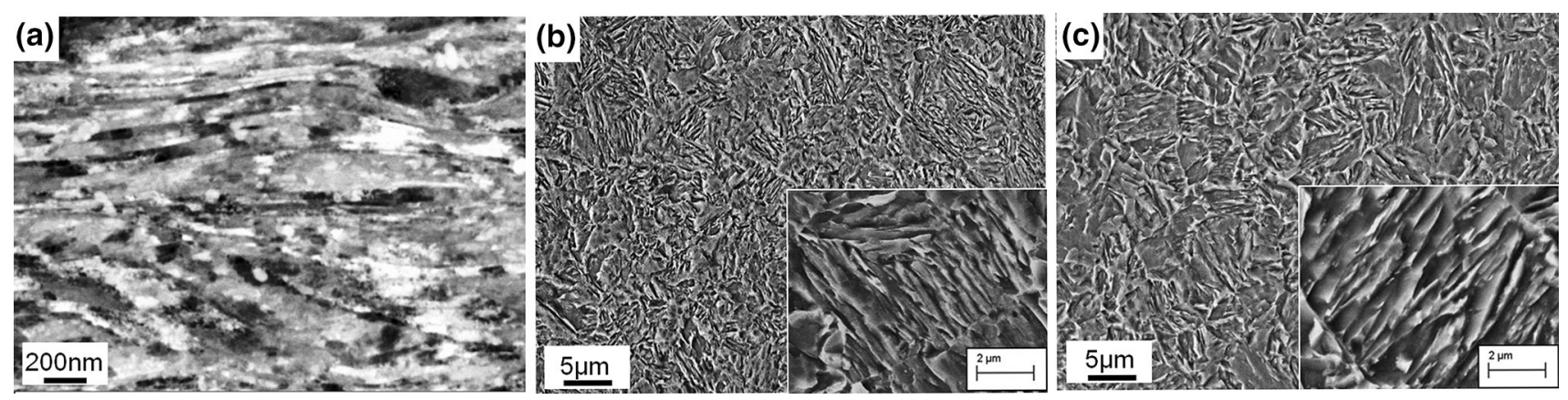

Fig. 3 SEM microstructures of the Nb-Mo microalloyed 6.5Mn steel: the as-cold rolled a, the Q \& $\mathrm{P}$ processed after austenitized at $\mathbf{b} 800{ }^{\circ} \mathrm{C}$; c $850{ }^{\circ} \mathrm{C}$ 

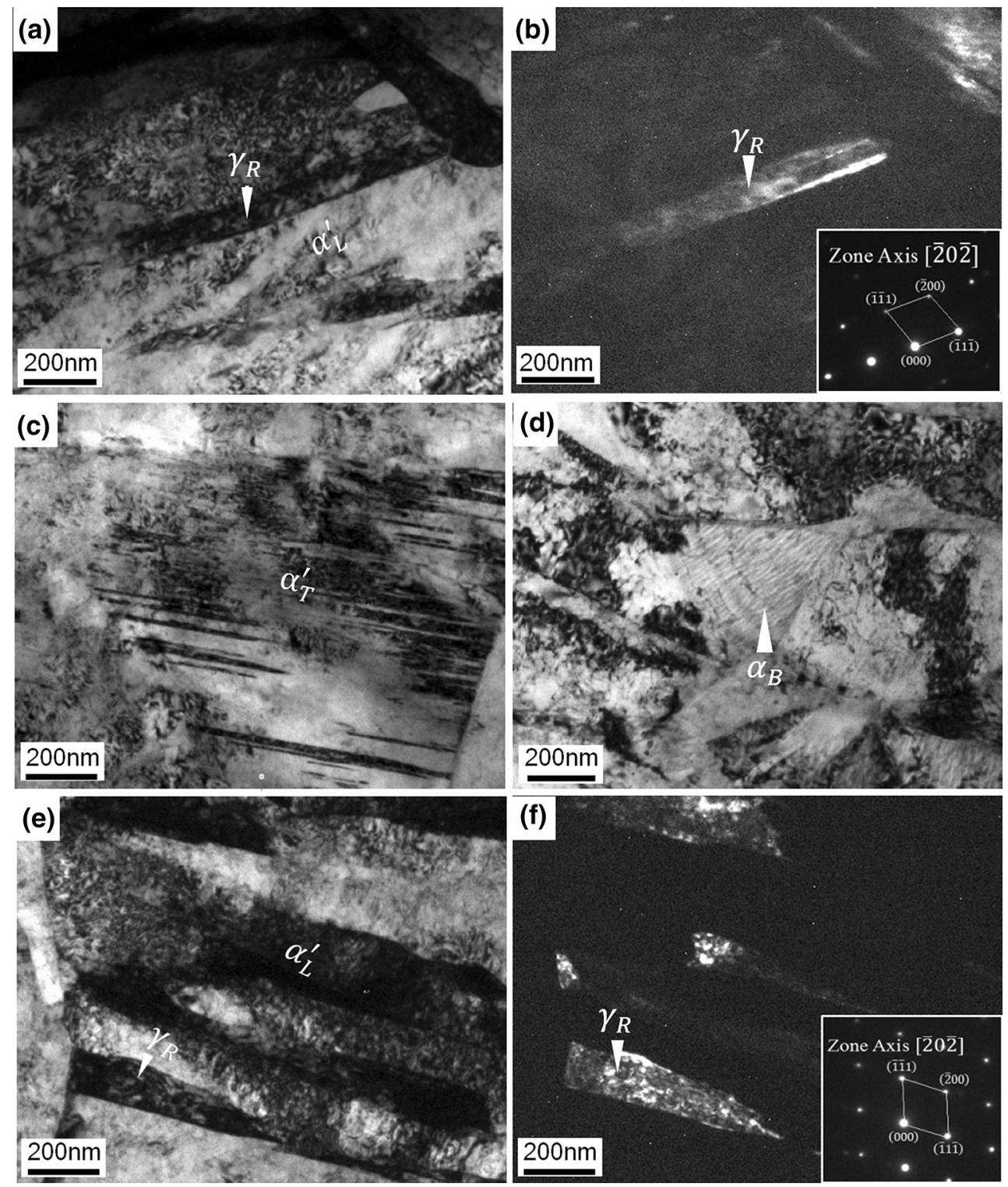

Fig. 4 TEM morphologies of the cold-rolled Nb-Mo microalloyed 6.5Mn steel treated by Q \& $\mathrm{P}$ with different austenization temperatures: a-c $800{ }^{\circ} \mathrm{C} ; \mathbf{d}-\mathbf{f} 850{ }^{\circ} \mathrm{C} ; \mathbf{b}, \mathbf{f}$ correspond to the dark field images of a, e, respectively. $\gamma_{\mathrm{R}}$ retained austenite; $\alpha_{\mathrm{B}}$ carbide-free bainite; $\alpha_{\mathrm{L}}^{\prime}$ lath martensite; $\alpha_{\mathrm{T}}^{\prime}$ twinning martensite

variation of $V_{\mathrm{R}}$ with increasing annealing time may be the combined effects of the following two aspects: (1) the volume fraction of reversed austenite increased, causing the increase in $V_{R}$; (2) the stability of reversed austenite decreased due to the dilute concentration of alloying elements in austenite and the coarsening microstructure, leading to the decrease in $V_{\mathrm{R}}$.

\subsection{Mechanical Properties}

The tensile properties for both IA and Q \& P samples are illustrated in Table 1. For IA samples, the ultimate tensile strength (UTS) ranged from 1086 to $1259 \mathrm{MPa}$, the yield strength (YS) from 843 to $1143 \mathrm{MPa}$. Both UTS and YS showed a fluctuating trend with the annealing time. The comprehensive tensile properties of samples were characterized by the product (PSE) of UTS and total elongation (EL). The PSE of IA samples first increased to $40.4 \mathrm{GPa} \%$ at approximately $30 \mathrm{~min}$ and then generally decreased with increasing annealing time. In contrast, the Q \& P samples exhibited both relatively higher UTS and YS, but very lower EL: when the austenization temperature increased from 800 to $850{ }^{\circ} \mathrm{C}$, the UTS decreased by about $180 \mathrm{MPa}$ and YS about $100 \mathrm{MPa}$, together with a slight increase in EL by $4 \%$. 


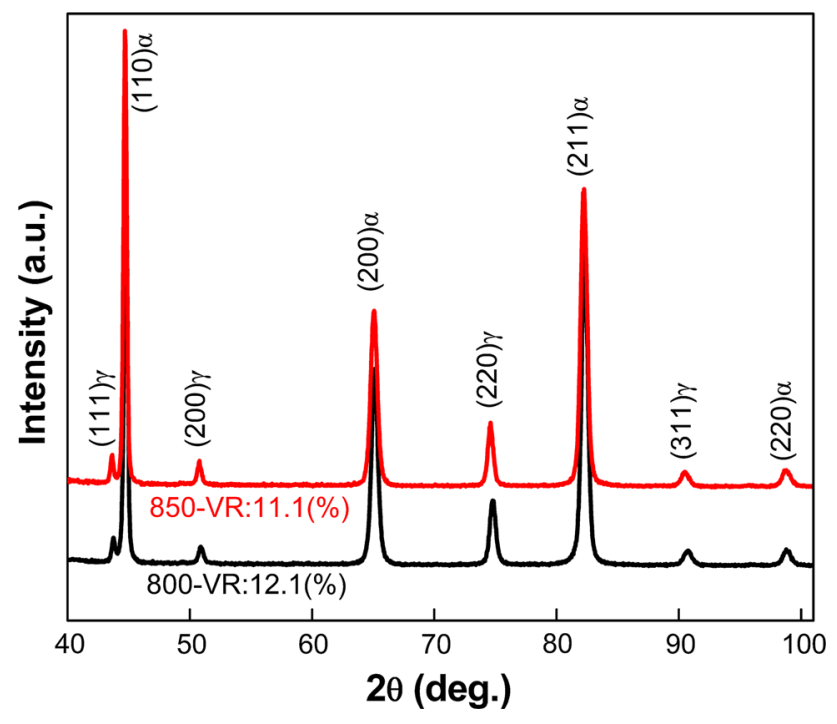

Fig. 5 X-ray diffraction spectra of the cold-rolled Nb-Mo microalloyed $6.5 \mathrm{Mn}$ steel treated by $\mathrm{Q} \& \mathrm{P}$ with different austenization temperatures

\section{Discussion}

\subsection{Effect of Heat Treatments on the Microstructural Evolution}

The microstructural evolution during IA process of the cold-rolled $\mathrm{Nb}-\mathrm{Mo}$ microalloyed $6.5 \mathrm{Mn}$ steel was quite different from that of $\mathrm{Q} \& \mathrm{P}$ process. As schematically illustrated in Fig. 8, the IA-processed microstructure consisted of ferrite and austenite, which were a result of competition of fcc reverse transformation and bcc dynamic recrystallization [17, 26]. Nakada et al. [27] computationally simulated the reverse transformation of $0.1 \mathrm{C}-5 \mathrm{Mn}$ steel, revealing that the $\alpha^{\prime} \rightarrow \gamma$ transformation was relatively fast, and thus the austenite fraction rapidly increased, eventually reaching an equilibrium, which was in good agreement with the present result in Fig. $7 \mathrm{~b}$. The rapid austenite formation was mainly attributed to the heavily deformed $\alpha^{\prime}$ by $70 \%$ reduction cold rolling, which could
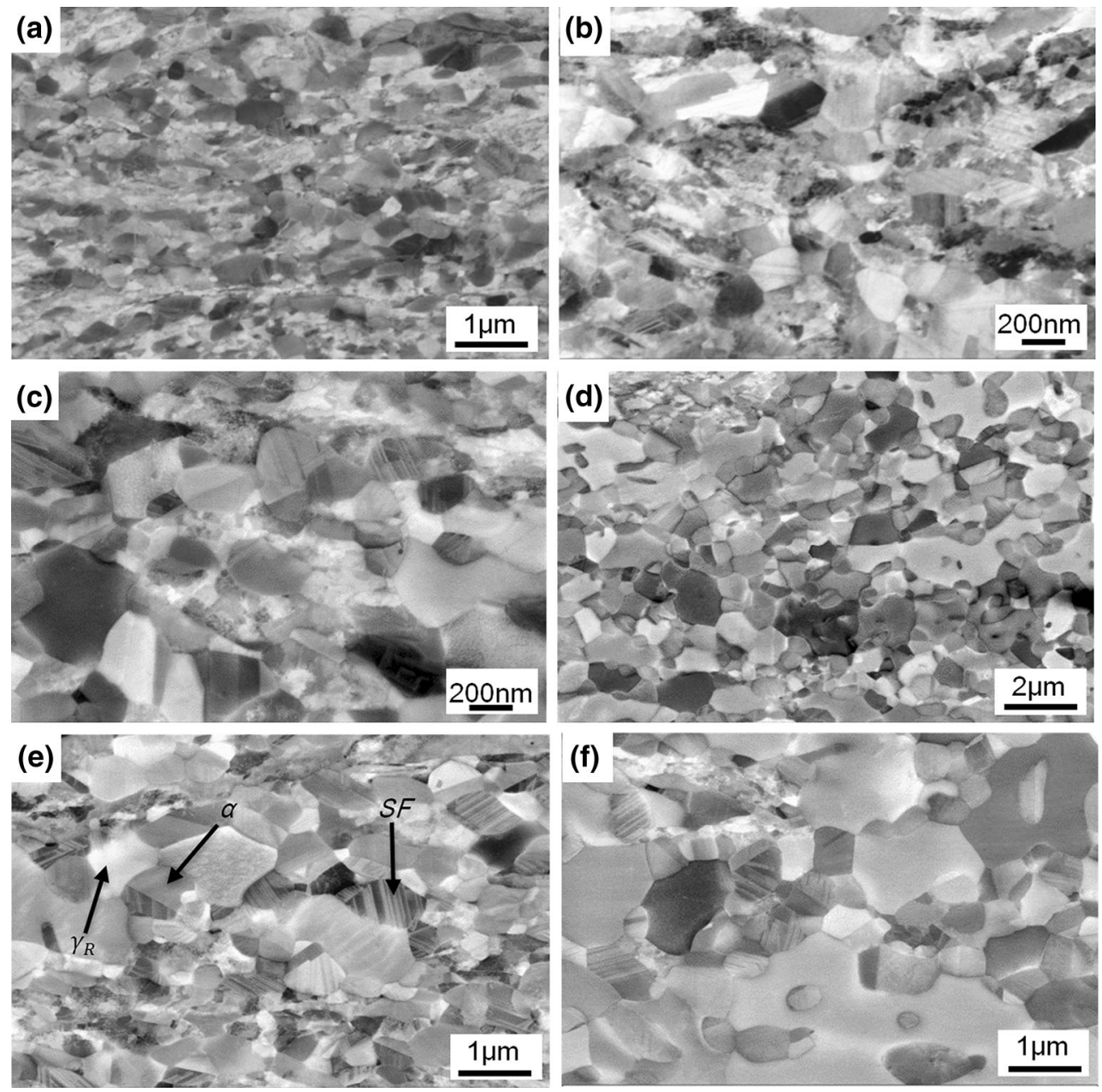

Fig. 6 SEM micrographs of the cold-rolled Nb-Mo microalloyed $6.5 \mathrm{Mn}$ steel treated by IA at $650{ }^{\circ} \mathrm{C}$ for different time: a $10 \mathrm{~min}$; b $30 \mathrm{~min}$; $\mathbf{c}$ $60 \mathrm{~min}$; d $180 \mathrm{~min}$; e $270 \mathrm{~min}$; $\mathbf{f} 360 \mathrm{~min} . \alpha$ ferrite; $\gamma_{\mathrm{R}}$ retained austenite; $S F$ stacking fault 

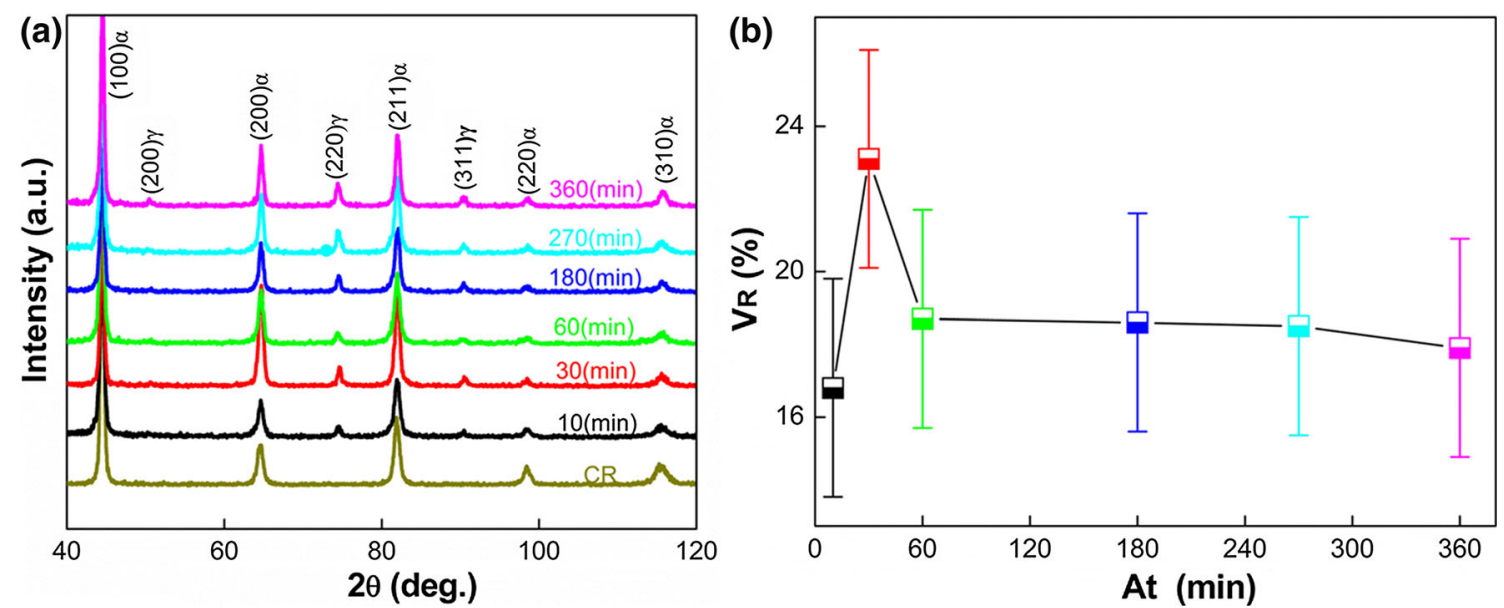

Fig. 7 a X-ray diffraction patterns; $\mathbf{b}$ the volume fraction of retained austenite $\left(\mathrm{V}_{R}\right)$ of the cold-rolled Nb-Mo microalloyed 6.5Mn steel treated by IA at $650{ }^{\circ} \mathrm{C}$ for different holding time

Table 1 Mechanical properties of the cold-rolled Nb-Mo microalloyed 6.5Mn steel for both IA and Q \& P processes

\begin{tabular}{|c|c|c|c|c|c|}
\hline Process & Parameter $\left(\min /{ }^{\circ} \mathrm{C}\right)$ & YS (MPa) & UTS (MPa) & $\mathrm{EL}(\%)$ & PSE $(\mathrm{GPa} \%)$ \\
\hline \multirow[t]{6}{*}{ IA } & 10 & 1143 & 1259 & 26.9 & 33.9 \\
\hline & 30 & 1097 & 1224 & 33.0 & 40.4 \\
\hline & 60 & 983 & 1184 & 30.0 & 35.5 \\
\hline & 180 & 863 & 1116 & 34.1 & 38.1 \\
\hline & 270 & 881 & 1242 & 30.1 & 37.4 \\
\hline & 360 & 911 & 1086 & 33.9 & 36.8 \\
\hline \multirow[t]{2}{*}{$\mathrm{Q} \& \mathrm{P}$} & 800 & 1150 & 1400 & 10.0 & 14.0 \\
\hline & 850 & 1050 & 1220 & 14.0 & 17.1 \\
\hline
\end{tabular}

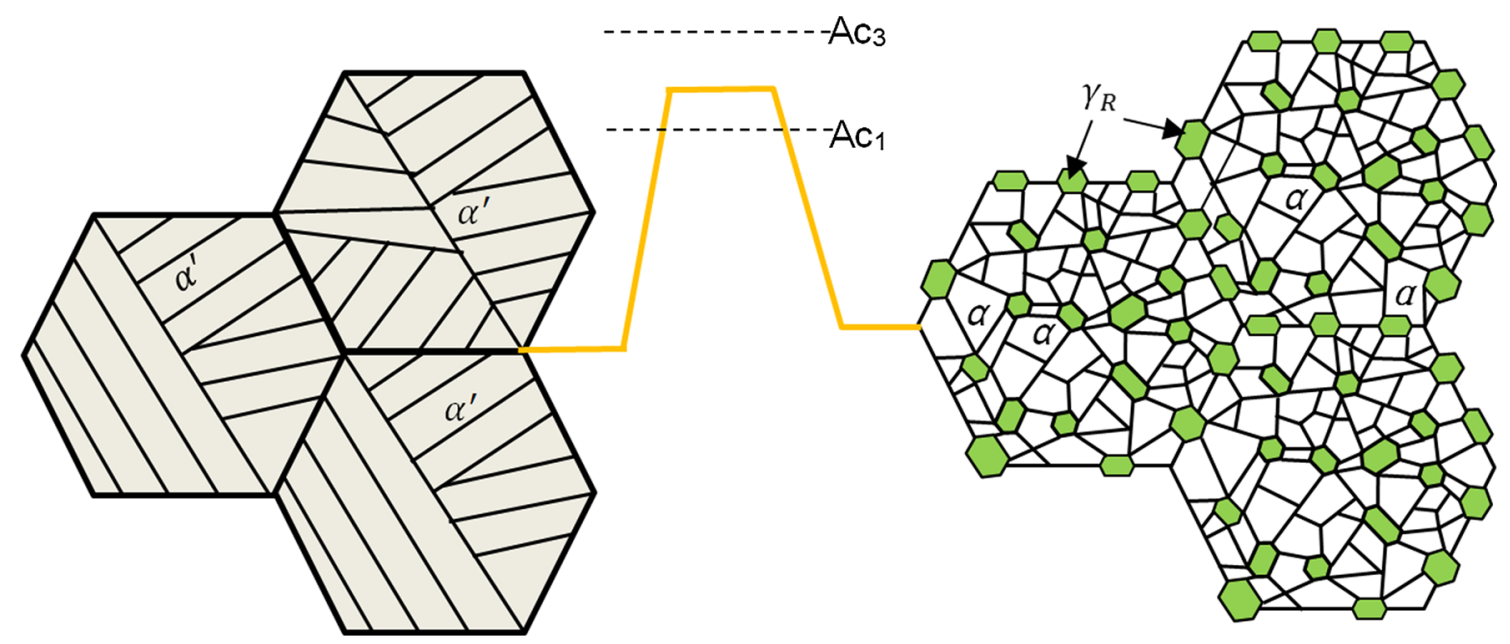

Fig. 8 Schematic illustrations of microstructural evolution of the cold-rolled Nb-Mo microalloyed 6.5Mn steel treated by IA. $\alpha^{\prime}$ deformed martensite; $\alpha$ ferrite; $\gamma_{R}$ retained austenite

provide a higher density of austenite nucleation sites. From Figs. 6 and 8, it can be found that nucleation of $\gamma$ phase occurred preferentially at grain boundaries or grainboundary triple points, which agreed with the result by Shi et al. [28].
More interestingly, the $\alpha^{\prime} \rightarrow \gamma$ transformation remained to room temperature even after cooling because of the sufficient thermal stability resulting from the solute partitioning (Mn and C) [29]. Meanwhile, the heavily deformed martensitic laths were subdivided into finer crystals with 


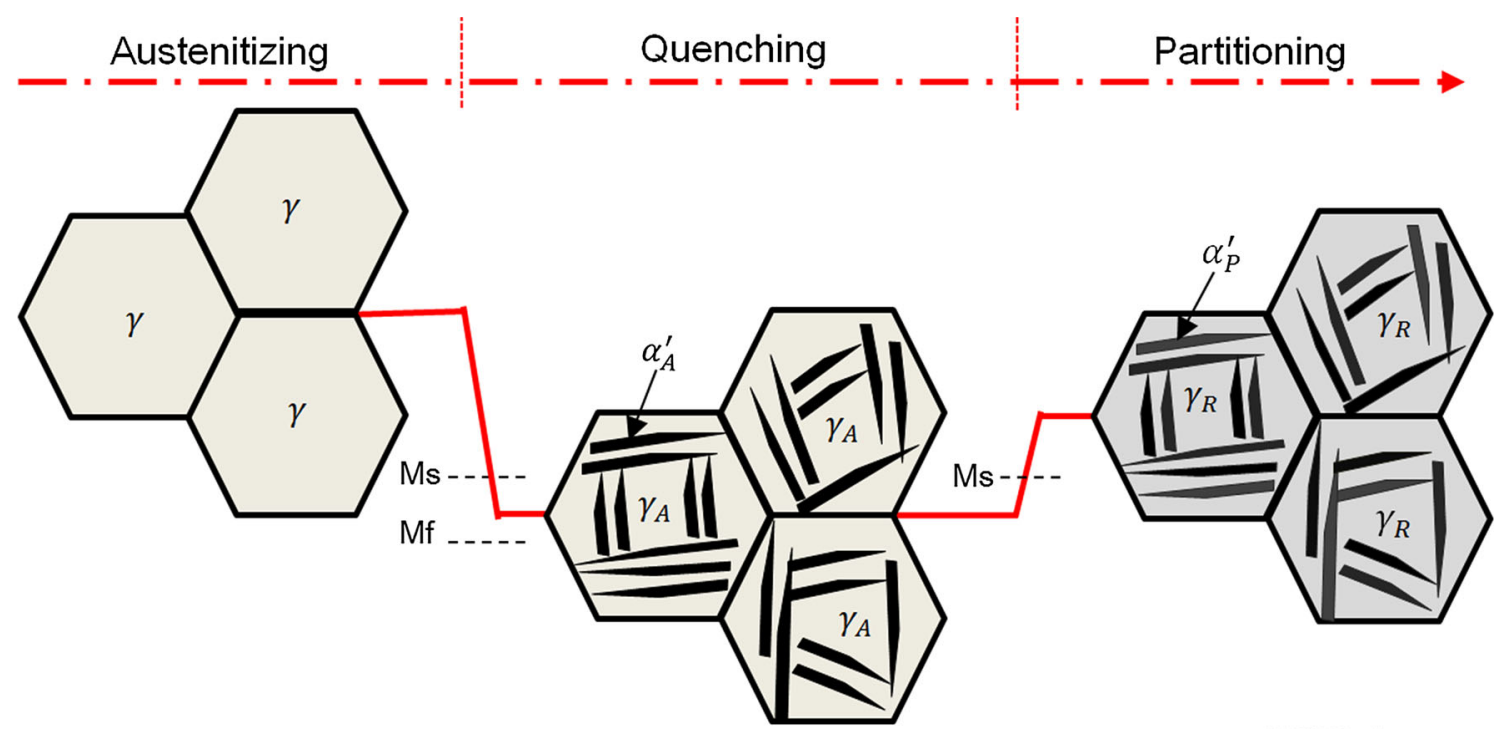

Fig. 9 Schematic illustrations of microstructural evolution of the cold-rolled Nb-Mo microalloyed 6.5Mn steel treated by Q \& P. $\gamma$ austenite; $\alpha_{\mathrm{A}}^{\prime}$ quenched martensite; $\gamma_{\mathrm{A}}$ quenched austenite; $\gamma_{\mathrm{R}}$ retained austenite; $\alpha_{\mathrm{P}}^{\prime}$ partitioned martensite

high density of dislocations, due to the recovery and recrystallization during subsequent IA process, and eventually caused the formation of new equiaxed ferrite grains with high-angle grain boundaries [17].

However, the experimental Q \& $\mathrm{P}$ process involved partially quenching to the temperature between martensitestart $\left(M_{\mathrm{s}}\right)$ and martensite-finish $\left(M_{\mathrm{f}}\right)$ temperatures, followed by partitioning above the $M_{\mathrm{s}}$. Thus, the deformed $\alpha^{\prime}$ microstructure was transformed to the mixture of the asquenched martensite $\alpha_{A}^{\prime}$, the retained austenite rich in carbon, which escaped from the supersaturated $\alpha_{A}^{\prime}$, and the partitioned martensite $\alpha_{P}^{\prime}[30,31]$, as detailed in Fig. 9.

In contrast, the fraction of retained austenite $V_{R}$ $(\sim 23 \%)$ obtained during IA was much higher than that ( $\sim 12.1 \%$ ) during $\mathrm{Q} \& \mathrm{P}$ process. This can be explained as followings: the diffusion of both $\mathrm{Mn}$ and $\mathrm{C}$ occurred from the deformed $\alpha^{\prime}$ to austenite during IA process [29, 32], whereas there was almost no Mn diffusion during $\mathrm{Q} \& \mathrm{P}$ process [22]. In addition, the diffusivity of $\mathrm{C}$ was also different due to the difference between the IA and partitioning temperatures. Both $\mathrm{Mn}$ and $\mathrm{C}$ are strong austenite stabilizing elements, thus leading to the higher $V_{\mathrm{R}}$ in IA.

\subsection{Effect of Heat Treatments on Mechanical Properties}

The significant difference in microstructural evolution during IA and $\mathrm{Q} \& \mathrm{P}$ processes of the cold-rolled $\mathrm{Nb}-\mathrm{Mo}$ microalloyed $6.5 \mathrm{Mn}$ steel led to the variation of final mechanical properties to some extent, as displayed in Table 2. The Q \& P steels showed slightly higher UTS and YS values but extremely lower EL values when compared to the IA-processed steels. Accordingly, the PSE values of IA samples (33.9-40.4 GPa\%) were much larger than those (14-17.1 GPa\%) of the Q \& P samples. According to the analysis in Sect. 4.1, the Q \& P samples exhibited relatively higher UTS than the IA samples, which was mainly attributed to the martensite matrix with high dislocation density, leading to high working hardening rate. The improved ductility of IA samples mainly benefited from the more sufficient TRIP effect due to the significantly higher $V_{\mathrm{R}}$. Moreover, the softer ferrite with low density of dislocations also contributed to the increase in tensile ductility due to the fact that the soft ferrite could undertake more plastic deformation than the hard martensite upon loading [33].

\subsection{Effect of Heat Treatments on Deformation Behavior}

As shown in Fig. 10, all IA tensile samples exhibited discontinuous yielding behavior, i.e., Lüders band propagation

Table 2 YPE of the cold-rolled Nb-Mo microalloyed 6.5Mn steel treated by IA

\begin{tabular}{lc}
\hline AT $(\min )$ & YPE $(\%)$ \\
\hline 10 & 11.46 \\
30 & 11.24 \\
60 & 9.72 \\
180 & 8.83 \\
270 & 7.29 \\
360 & 1.82 \\
\hline
\end{tabular}



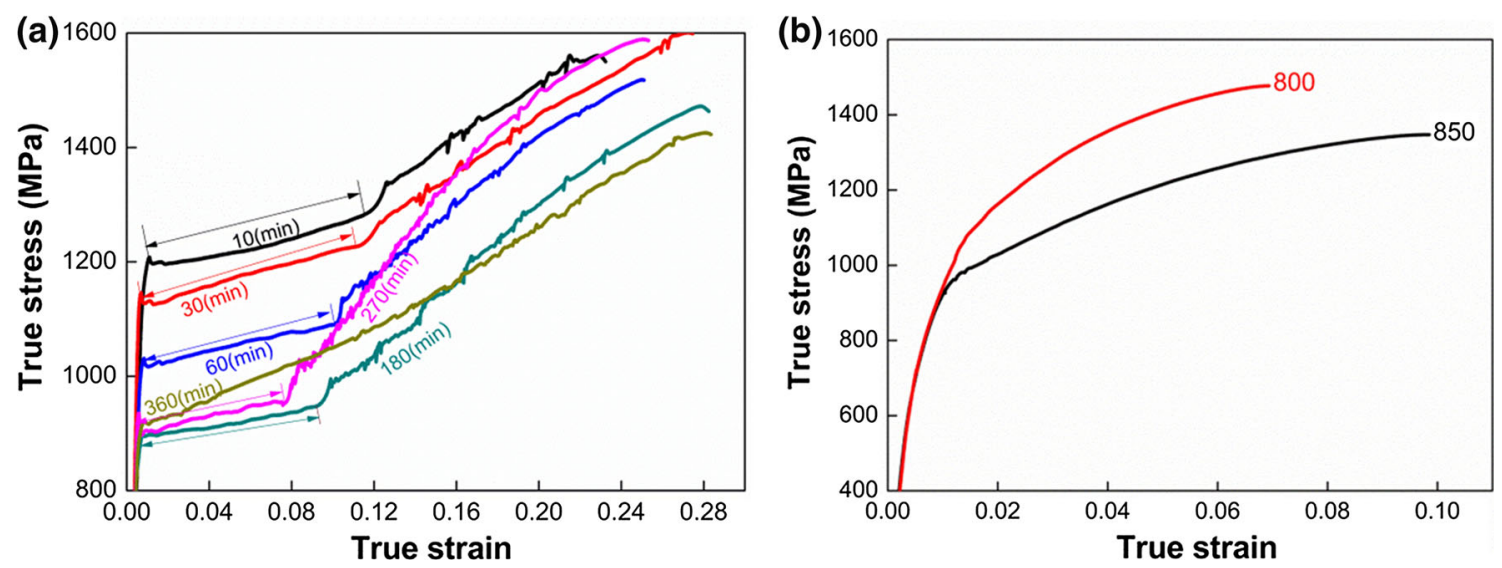

Fig. 10 True stress-strain curves of the cold-rolled Nb-Mo microalloyed 6.5Mn steel after IA a, Q \& P b processes
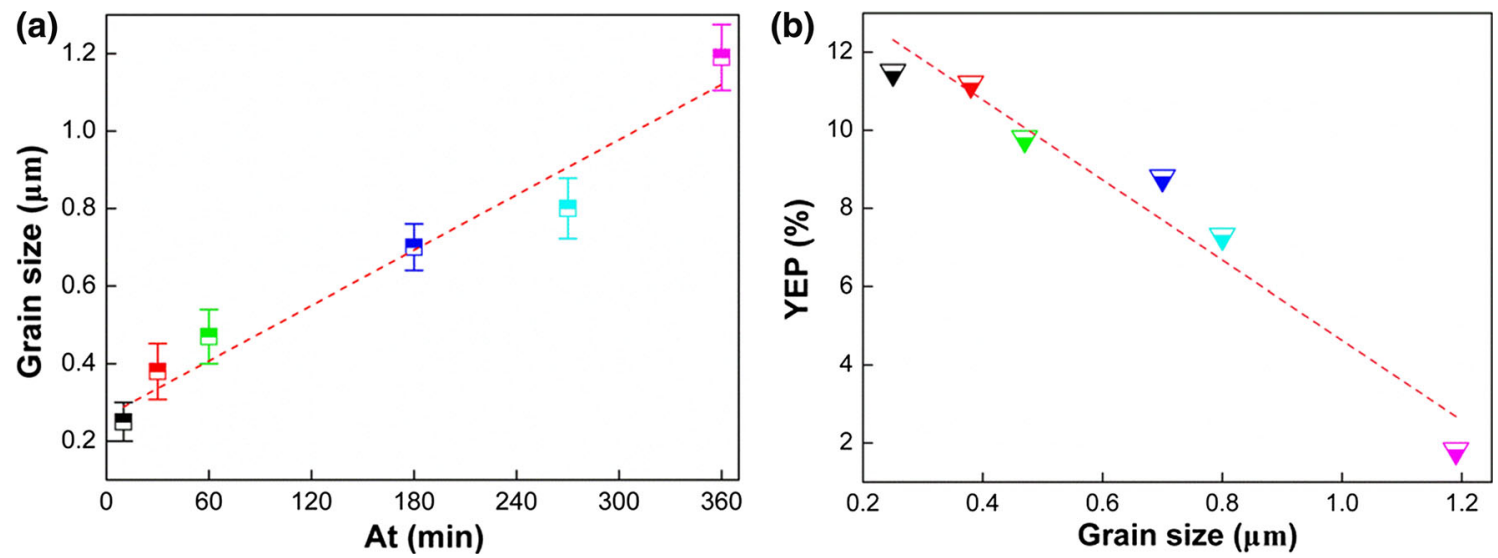

Fig. 11 a Grain size versus annealing time; b yield point elongation versus grain size of the cold-rolled Nb-Mo microalloyed 6.5Mn steel treated by IA

(LBP) appeared, whereas both Q \& P samples showed continuous yielding behavior during plastic deformation. The LBP phenomenon has been commonly reported in the cold-rolled medium Mn steel treated by IA, which was considered as a result of local deformation in ferrite with low density of dislocations [33, 34]. In the present work, the discrepancy in LBP between IA and Q \& $\mathrm{P}$ samples may be caused by the difference in initial deformation cites. For the IA samples, the plastic deformation occurred firstly in the soft ferrite, which accumulated more strain before TRIP effect, leading to the local deformation. However, in the case of $\mathrm{Q} \& \mathrm{P}$, the plastic deformation occurred firstly in $\gamma_{R}$, resulting in the continuous yielding behavior due to the TRIP effect.

Further study on the LBP of IA was performed to clarify the influence of annealing time on yield point elongation (YPE). The specific values of YPE with annealing time are illustrated in Table 2. It can be seen that the YPE decreased with increasing the annealing time: (1) as the annealing time was less than $30 \mathrm{~min}$, the YPE was relatively high $(>10 \%)$; (2) with further increasing annealing time, the
YPE dramatically decreased and only reached $1.83 \%$ for the 360 min annealed sample. This implied that the YPE phenomenon was closely related to the grain size, which has been reported by Hayes et al. [35] and Barnett et al. [36].

A relationship between YPE and propagation rate of Lüders band was described by $\mathrm{Hu}$ [37] as follows.

When a Lüders band crossed a length $\delta l$ of sample in time $\delta t$, then

$V_{\mathrm{L}}=\delta l / \delta t$,

where $V_{\mathrm{L}}$ is the propagation rate of Lüders band.

Considering that the tensile testing was performed at a constant rate, the length in increment of samples in time $\delta t$ should be equal to the displacement moved by testing machine. This could be expressed as:

$\left(1+\varepsilon_{\mathrm{L}}\right) \delta l-\delta l=V_{\mathrm{C}} \delta t$,

where $\varepsilon_{\mathrm{L}}$ is the Lüders strain and $V_{\mathrm{C}}$ is the velocity of the testing machine.

Thus, Eq. (6) could be converted into: 
$\varepsilon_{\mathrm{L}}=V_{\mathrm{C}} / V_{\mathrm{L}}$.

According to Eq. (7), qualitative analysis of the relationship between Lüders $\varepsilon_{\mathrm{L}}$ strain and propagation rate of Lüders band $V_{\mathrm{L}}$ was given here.

It is easy to understand that the grain size increased with increasing annealing time (Fig. 11a). Grain coarsening led to the higher propagation rate of Lüders band, due to the decreasing number of grain boundaries in unit area, which could serve as obstacles for LBP, thus resulting in the relatively lower YPE, based on Eq. (7). Further analysis of the dependence of YPE on grain size (Fig. 11b) demonstrated that the YPE was in the inverse proportional relationship to the average grain size, which agreed well with the above theoretical analysis.

\section{Conclusions}

The IA and Q \& P processed Nb-Mo microalloyed 6.5Mn samples were used to compare the differences in transformation behavior, microstructural evolution and mechanical properties. The obtained results are as follows:

1. The $\mathrm{Q} \& \mathrm{P}$ microstructure was characterized by lath martensite, twinning martensite, carbide-free bainite and some amount of retained austenite (11-12\%).

2. The IA microstructure consisted of equiaxed ferrite and retained austenite, and the volume fraction of retained austenite ranged from $17 \pm 3$ to $23 \% \pm 3 \%$ with varying annealing time, which was a result of competition of fcc reverse transformation and bcc dynamic recrystallization.

3. The Q \& P samples showed slightly higher UTS and YS values but extremely lower EL values than those IA samples, and thus the PSE of Q \& P samples (14-17.1 GPa\%) were much lower than those (33.9-40.4 GPa\%) of IA samples.

4. All IA samples exhibited discontinuous yielding behavior upon loading, i.e., Lüders band propagation appeared, whereas both Q \& P samples had continuous yielding behavior during plastic deformation. The yield point elongation of IA samples was in the inverse proportional relationship to the average grain size.

Acknowledgements This work was financially supported by the National Natural Science Foundation of China (No. 51401050) and the Fundamental Research Funding for the Central Universities (No. N160204001). This work was also supported by the Australian Research Council (ARC) Laureate Fellowship (Prof. Hodgson). Part of work was carried out with the support of the Deakin advanced characterization facility.

\section{References}

[1] A.S. Hamada, L.P. Karjalainen, M.C. Somani, Mater. Sci. Eng. A 467, 114 (2007)

[2] E.B. Pan, H.S. Di, G.W. Jiang, C.R. Bao, Acta Metall. Sin. (Engl. Lett.) 27, 469 (2014)

[3] L.Q. Chen, Y. Zhao, X.M. Qin, Acta Metall. Sin. (Engl. Lett.) 26, 1 (2013)

[4] R.L. Miller, Metall. Mater. Trans. A 3, 905 (1972)

[5] J. Han, S.J. Lee, J.G. Jung, Y.K. Lee, Acta Mater. 78, 369 (2014)

[6] S. Lee, B.C. De Cooman, Metall. Mater. Trans. A 44, 5018 (2013)

[7] J.M. Jang, S.J. Kim, N.H. Kang, K.M. Cho, D.W. Suh, Met. Mater. Int. 15, 909 (2009)

[8] W.Q. Cao, C. Wang, J. Shi, M.Q. Wang, W.J. Hui, H. Dong, Mater. Sci. Eng. A 528, 6661 (2011)

[9] D.W. Suh, S.J. Kim, Scr. Mater. 126, 63 (2017)

[10] J. Shi, X. Sun, M. Wang, W.Q. Cao, Scr. Mater. 63, 815 (2010)

[11] H. Aydin, E. Essadiqi, I.H. Jung, S. Yue, Mater. Sci. Eng. A 564, 501 (2013)

[12] J.G. Speer, D.K. Matlock, B.C.D. Cooman, J.G. Schroth, Acta Mater. 51, 2611 (2003)

[13] C.Y. Wang, Y.J. Zhang, W.Q. Cao, J. Shi, H. Dong, Sci. China Technol. Sci. 55, 1844 (2012)

[14] D.V. Edmonds, K. He, F.C. Rizzo, D.K. Matlockd, G. Speer, Mater. Sci. Eng. A 25, 438 (2006)

[15] H.J. Hu, G. Xu, L. Wang, G.H. Liu, Mater. Des. 84, 95 (2015)

[16] N. Isasti, D. Jorge-Badiola, M.L. Mitra, P. Uranga, Metall. Mater. Trans. A 44, 3552 (2013)

[17] M.H. Cai, Z. Li, Q. Chao, P.D. Hodgson, Metall. Mater. Trans. A 45, 5624 (2014)

[18] ASTM E8/E8M-2013, Standard Test Methods for Tension Testing of Metallic Materials

[19] B.D. Cullity, S.R. Stock, Elements of X-Ray Diffraction (Prentice Hall, New Jersey, 2001), p. 347

[20] R.M. Wu, W. Li, C.L. Wang, Y. Xiao, L. Wang, X.J. Jin, Acta Metall. Sin. (Engl. Lett.) 28, 368 (2015)

[21] I.B. Timokhina, P.D. Hodgson, E.V. Pereloma, Metall. Mater. Trans. A 35, 2331 (2004)

[22] E.J. Seo, L. Cho, B.C. DeCooman, Acta Mater. 107, 354 (2016)

[23] F.Y. Wang, Y.F. Zhu, H.H. Zhou, B.Z. Jiang, Sci. China Technol. Sci. 56, 1847 (2013)

[24] S.J. Lee, C.J. Tyne, Metall. Mater. Trans. A 43, 422 (2012)

[25] C. Capdevila, F.G. Caballero, ISIJ Int. 42, 894 (2002)

[26] J. Shi, H.F. Xu, J. Zhao, W.Q. Cao, C. Wang, C.Y. Wang, J. Li, H. Dong, Acta Metall. Sin. (Engl. Lett.) 25, 111 (2012)

[27] N. Nakada, K. Mizutani, T. Tsuchiyama, S. Takaki, Acta Mater. 65, 251 (2014)

[28] J. Shi, J. Hu, C. Wang, C.Y. Wang, H. Dong, W.Q. Cao, J. Iron. Steel Res. Int. 21, 208 (2014)

[29] S.J. Lee, S. Lee, B.C. DeCooman, Scr. Mater. 64, 649 (2011)

[30] J.G. Speer, E.D. Moor, K.O. Findley, D.K. Matlock, B.C. De Cooman, Metall. Mater. Trans. A 42, 3591 (2011)

[31] M.J. Santofimia, L. Zhao, J. Sietsma, Metall. Mater. Trans. A 42, $3620(2011)$

[32] S. Lee, S.J. Lee, S.S. Kumar, K. Lee, B.C. De Cooman, Metall. Mater. Trans. A 42, 3638 (2011)

[33] J. Han, S.J. Lee, J.G. Jung, Y.K. Lee, Acta Mater. 78, 369 (2014)

[34] Y.G. Deng, H.S. Di, J.C. Zhang, Acta Metall. Sin. (Engl. Lett.) 28, 1141 (2015)

[35] J.S. Hayes, R. Keyte, P.B. Prangnell, Mater. Sci. Technol. 16, 1259 (2000)

[36] R.M. Barnett, D.M. Nave, A. Ghaderi, Acta Mater. 60, 1433 (2012)

[37] H. Hu, Metall. Mater. Trans. A 14, 85 (1983) 